\title{
ILCEA
}

Revue de l'Institut des langues et cultures

d'Europe, Amérique, Afrique, Asie et Australie

9 | 2007

L'implicite et les écrits de l'entreprise

\section{Le sport et l'implicite de la prédation}

\section{Christian Leblond}

\section{OpenEdition}

Journals

Édition électronique

URL : http://journals.openedition.org/ilcea/668

DOI : 10.4000/ilcea.668

ISSN : 2101-0609

\section{Éditeur}

UGA Éditions/Université Grenoble Alpes

\section{Édition imprimée}

Date de publication : 15 décembre 2007

Pagination : $99-113$

ISBN : 978-2-84310-104-5

ISSN : 1639-6073

\section{Référence électronique}

Christian Leblond, « Le sport et l'implicite de la prédation », ILCEA [En ligne], 9 | 2007, mis en ligne le 09 juin 2010, consulté le 01 mai 2019. URL : http://journals.openedition.org/ilcea/668 ; DOI : 10.4000/ ilcea.668 


\title{
Le sport et l'implicite de la prédation
}

\author{
Christian LEBLOND \\ Université Stendhal-Grenoble 3
}

\begin{abstract}
RÉSUMÉ
Les entreprises qui fabriquent et commercialisent des produits sportifs mettent en général l'accent sur l'aspect consensuel du sport, décrit au niveau explicite comme un loisir de masse, paisible et relativement anodin. En appliquant la théorie institutionnelle de l'économiste T. Veblen, théoricien de la classe de loisir, cet article vise à souligner la complexité de la perception du sport en réintroduisant un contenu implicite souvent perceptible en arrièreplan. Le versant obscur du sport est remis dans sa perspective historique afin de mettre en évidence la permanence d'une mémoire collective archaïque, laquelle met en forme certaines de nos perceptions. Le modèle de Veblen peut contribuer à une meilleure compréhension des phénomènes mis en œuvre lors de l'achat d'un équipement sportif. Cette étude ne cherche pas à invalider le postulat de rationalité du consommateur mais à montrer que l'approche institutionnelle permet d'élaborer un modèle plus pertinent, éclairant notamment la politique de prix des équipementiers.
\end{abstract}

\section{ABSTRACT}

Firms manufacturing and marketing sports equipment generally emphasize the consensual side of sports, explicitly described as a fairly innocuous and peaceful form of mass leisure. Applying T. Veblen's institutionalist theory of the leisure class, this article aims at showing that the perception of sports is more complex because an implicit content is always present in the background. The dark side of sports is put in a historical perspective to highlight the way an archaic form of collective memory subliminally shapes our perceptions. The Veblen model can contribute to a fuller and deeper understanding of the motivations for buying sports equipment. It does not disprove the postulate of the consumer's rationality, but may show that the institutionalist approach provides us with a more relevant model to explain why some goods sell at a premium on a mass market.

Mots-clés

Veblen, marketing du sport, classe de loisir et sport, violence et sport.

Key words

Veblen, marketing of sport, leisure class and sport, violence and sport. 


\section{Un projet de lecture véblénienne}

Les entreprises qui ont trait au sport commercialisent des produits d'une nature particulière, qui permettent en principe une activité physique. Ces produits - des vêtements techniques ou de l'équipement - sont des accessoires choisis par un consommateur dans le but précis de pratiquer un sport. Mais il est évident qu'un grand nombre de ces produits ont des fonctions dérivées, et peuvent apparaître comme la promesse d'une activité, un emblème d'appartenance à un groupe de pratiquants ou un mode d'affichage de son style de vie. Les usages symboliques semblent parfois constituer la destination principale de ce type de biens. Dans ce détournement extrêmement fréquent, le produit procure une satisfaction à l'usager - acheteur plutôt que pratiquant - sans nécessairement servir sur le terrain. Cas extrême, les accessoires du supporter sont les oripeaux dont il convient de s'affubler pour regarder l'action du haut des gradins afin de transformer magiquement son inaction en un effort collectif, dont on s'attribuera, le cas échéant, les mérites. Le spectateur utilise ces accessoires pour afficher sa proximité avec les exploits réalisés, et capter ainsi une fraction du prestige des joueurs. Une telle consommation consiste donc à s'afficher socialement plus qu'à agir physiquement. L'accessoire de sport arboré hors du terrain renvoie aux exploits passés et sans doute futurs, et indique que dans l'intervalle, le sportif s'adonne au loisir.

Ce type de comportement qui est l'affichage d'une oisiveté parfois passablement tapageuse après la prouesse, renvoie aux concepts développés par Thornstein Veblen, dans sa Théorie de la Classe de Loisir ${ }^{1}$. Cet économiste américain (1857-1929), un des fondateurs du courant «institutionnaliste ${ }^{2} \gg$, explique, dans son célèbre ouvrage, les comportements économiques sur d'autres bases que l'individualisme méthodologique et le postulat de rationalité de l'bomo economicus.

Pour les économistes classiques et néo-classiques, le sujet économique est un calculateur avisé qui cherche à maximiser l'utilité dans toutes ses actions. Veblen constate que cette vision ne rend pas compte de nombreux comportements comme la consommation de produits de luxe dont les prix exorbitants devraient entraîner une demande proche de zéro. Pour les institutionnalistes, les choix sont dictés par le groupe et ses conceptions du prestige, façonnés au cours du passé pré-capitaliste de l'histoire humaine. La sémiotique de Veblen met l'accent sur le loisir et la consommation ostentatoire, moyens d'afficher son appartenance à la classe supérieure, celle qui, pour simplifier, pratique d'abord la prédation

1. Par la suite, l'ouvrage sera désigné par TCL.

2. Les historiens de la pensée économique, de Spindler à Pressman en passant par Heilbroner, situent Veblen dans la mouvance de Marx. 
en accomplissant des exploits (prowess), puis expose ses trophées en menant une vie de loisir. Aux stades plus récents, moins barbares au sens de Veblen, «l'émulation pécuniaire ${ }^{3}$ » prend la suite des rivalités plus physiques, et le gaspillage ostentatoire d'argent (conspicuous waste) permet d'afficher son statut social. De là découle, chez Veblen, un système de la mode, une théorie de l'éducation supérieure, mais pas à proprement parler de théorie du sport.

Pourtant, le marketing sportif joue sur l'ambiguité des produits offerts au consommateur. Le message explicite met en avant l'exercice salutaire, l'effort individuel constant pour accéder à la performance, et un idéal de camaraderie qui s'incarne dans «l'esprit d'équipe» et le fair play - le fameux esprit sportif. Mais ce discours hygiéniste et moralement inattaquable coexiste avec des strates beaucoup moins explicites du phénomène, que nous essaierons de faire affleurer. Cet implicite du sport obéit à une logique qui gagne à être appréhendée à partir de la théorie de la classe de loisir. Une grille de lecture véblénienne sur le sujet peut rendre compte, au moins partiellement, des caractères paradoxaux de la consommation et de la promotion des articles vendus sous le logo des grandes marques sportives. Cette approche véblénienne de l'économie du sport s'appuiera sur la théorie de la classe de loisir sans s'interdire de recourir au second Veblen, le théoricien de la firme capitaliste qui publie en 1904 The Theory of Business Enterprise ${ }^{4}$.

Tout lecteur attentif de Veblen a l'habitude de trouver le mot sport dans la liste des activités caractéristiques de la classe dominante. Dès les premières pages, nous trouvons la liste des activités de loisir que sont «la guerre, la politique, les sports, l'étude et les fonctions religieuses» (Veblen, TCL, p. 3). Dans la division du travail moderne, entre classe laborieuse et classe de loisir, le sport est donc une activité «non industrielle» au même titre que la religion ou la politique, et les sportifs se retrouvent en compagnie des prêtres et des chefs de guerre. Mais les valeurs du sport affichées de façon explicite de nos jours sont plutôt pacifiques et démocratiques.

\section{Le sport aux deux visages}

La pratique sportive permet d'oublier les clivages sociaux, politiques ou religieux et constitue un dispositif d'intégration sociale classique. Les discours explicites sur le sport relèvent bien souvent du politiquement correct et le héros, celui qui triomphe de l'adversaire, voire le met à mort de

3. Titre du chapitre 2 de la Classe de Loisir.

4. Par la suite, $T B E$. 
façon symbolique, finit par illustrer des valeurs hautement consensuelles. Le légendaire Yogi Berra ${ }^{5}$, par exemple, donne son nom à un musée dont la mission est «de préserver et de promouvoir les valeurs de respect, de sportivité (sportsmanship), de justice sociale et d'excellence au moyen de programmes et d'expositions intégrateurs (inclusive), respectant la diversité culturelle et basés sur les sports».

Chez les équipementiers sportifs, le ton ne varie guère, et leurs sites web déclinent sous toutes les formes possibles le thème de l'entreprise citoyenne et responsable. Les grands acteurs du secteur, Adidas, Nike, Puma et Reebok ${ }^{6}$, chacun dans un style qui lui est propre, communiquent par des discours dont la tonalité d'ensemble et le contenu restent extraordinairement similaires. Violemment mis en cause dès la fin des années quatre-vingt-dix par Naomi Klein et Michael Moore - pour citer les noms les plus connus - sur des thèmes dévastateurs comme le travail des enfants chez les sous-traitants asiatiques ou l'écologie ${ }^{7}$, les firmes réagissent en moralisant leurs pratiques et en communiquant massivement sur leurs efforts dans ces domaines.

Adidas se prête à l'exercice en détaillant les initiatives prises pour le développement durable :

For the Adidas Group, being a global leader in the sporting goods industry means improving working conditions in our suppliers' factories and reducing our environmental impact as a business ${ }^{8}$.

Le groupe Reebok s'est, pour sa part, doté dès 1986 d'une fondation charitable qui distribue des dons selon «les principes résumés dans la Déclaration Universelle des Droits de l'Homme ${ }^{9} \gg$. Pour ne pas être en reste, la fondation Nike se fixe comme but d'aider «les jeunes filles qui sont le plus dans le besoin sur la planète ${ }^{10} \gg$.

Naturellement, la structure de ces grandes firmes-réseaux, typiques de l'économie mondialisée, n'est pas affectée en profondeur par cette offensive de communication. La révolte contre le logo n'a pas modifié la donne, et les critiques ont beau jeu d'évoquer l'hypocrisie de ces grands groupes. Il serait facile de développer une autre thématique de la prédation, qui insisterait sur l'exploitation de la main d'œuvre des pays du sud

5. L'un des plus célèbres joueurs de baseball, étoile des New York Yankees. Le musée qui porte son nom est situé à l'université Monclair dans le New Jersey : voir site du Yogi Berra Museum.

6. Le secteur des équipementiers est à la fois plus vaste et plus concentré, notament depuis l'OPA amicale de Adidas sur Reebok en août 2005.

7. La sous-traitance est dénoncée comme induisant les pays du sud à pratiquer le dumping écologique dans le discours altermondialiste et néo-protectionniste.

8. Voir site Adidas pour l'engagement sur le développement durable.

9. Voir le site de la RHRF (Reebok Human Rights Foundation)

10. C'est-à-dire typiquement la main d'œuvre de ses sous-traitants : voir le site Nike. 
et le tourbillon des fusions-acquisitions qui rythme le marché boursier. Mais ces phénomènes, bien que primordiaux et remarquablement analysés par Veblen en son temps, se déroulent à notre avis en dehors du champ de la conscience archaïque des consommateurs, et ne contribuent que marginalement aux mécanismes de fascination qui nous occupent ici.

Paradoxalement, cette offensive contre le marketing, voile bien peu opaque jeté sur des pratiques répréhensibles, redonne l'avantage à d'autres entreprises, perçues comme plus humaines, alors qu'elles sont tout aussi mondialisées, comme le groupe Columbia. La figure iconique de sa dirigeante «Mother Boyle», dont le mauvais caractère presque légendaire est intégré aux campagnes de publicité, contribue à rendre sympathique ce groupe, situé à Portland, Oregon comme Nike ${ }^{11}$. Même dans ce cas, la grille de Veblen permet de formuler l'opposition entre l'imaginaire héroïque de l'exploit sportif et un discours explicitement méritocratique, voire fortement coloré par l'éthique protestante du travail, que l'on retrouve dans un des préceptes favoris de «Mother Boyle : «Early to bed, early to rise, work like hell and advertise ${ }^{12} »$.

En tant que phénomène social, le sport figure de nos jours en bonne place dans les valeurs de la société de consommation, mais prétend participer à la marche vers le progrès des esprits. Pascal Bruckner reprend l'idée d'Alain Ehrenberg qui décrit avec un certain optimisme «[les] stades sans dieux» dans lesquels «le sport rend parfois les masses folles, [...] les met en extase ou en furie [...] mais [constitue] un vecteur de modernisation idéologique [qui] fait fonctionner les valeurs de la démocratie $^{13} \gg$. En somme, si la foule des spectateurs entre en ébullition devant les exploits de ses héros, ce serait pour mieux faire fonctionner le creuset démocratique et finalement égalitaire. L'ambition d'un tel rassemblement, comme le note Bruckner avec une certaine distance, est de «purger l'homme européen de son fond barbare [par] la publicité, les médias, la consommation $\gg$ (Bruckner, p. 94).

Mais refuser de prendre en compte un autre visage du sport n'est pourtant pas sans danger pour la compréhension du phénomène. S'en tenir exclusivement à l'explicite lumineux et démocratique, c'est se priver d'instruments d'analyse fournis par les théoriciens du marketing à partir de prémisses institutionnalistes revisitées.

11. La brutalité bon enfant assumée et même revendiquée par le manager rappelle irrésistiblement la Tante dans le célèbre et classique comic des Katzenjammer Kids (origine germanique, le fils Tim rudoyé, etc.)

12. Voir l'article sur le site de la Billings Gazette.

13. Bruckner cite Ehrenberg mais prend ses distances avec sa vision du désenchantement du sport. 
En effet, la théorie institutionnaliste permet de mettre en place des hypothèses sur le comportement du consommateur, pour qui l'appartenance au groupe et l'adhésion à son système de prestige et de distinction est un déterminant fondamental. Ces hypothèses sont peut-être discutables, mais elles sont confirmées de manière presque empirique par les tenants du marketing «tribal». Celui-ci consiste à découper le marché non selon les catégories socio-professionnelles habituelles, mais en tribus. Il reprend les caractéristiques du groupe ancestral pour dessiner les contours d'un groupe cible susceptible de consommer un type de produit précis. Le «totem», c'est-à-dire l'animal qui est l'ancêtre mythique du groupe, explique-t-on dans ces cours de marketing, correspond aux symboles et aux marques. Naturellement, la tribu Harley-Davidson n'intéresserait pas nécessairement Levi-Strauss, mais la transposition ainsi effectuée nous oriente vers un examen attentif des symboles dont se servent les équipes et les équipementiers pour communiquer.

\section{Implicite et mémoire}

L'hypothèse de Veblen émet l'idée que l'imaginaire du sport puise ses racines dans un terreau primitif, aristocratique, voire chamanique, qui se perçoit confusément sous des dehors relativement anodins. Veblen remarque que les animaux de proie sont extrêmement fréquents dans les écussons de l'aristocratie, avec une profusion de dragons, de lions et d'aigles en tout genre ${ }^{14}$. L'héraldique du sport, les emblèmes et les noms des équipes, obéit à la même tendance. Les rugbymen de l'équipe britannique sont, par exemple, des lions, ce qui n'est guère surprenant, vu l'omniprésence des armes royales sur divers produits, mais le football (américain) et le base-ball du Nouveau Monde fournissent un grand nombre d'illustrations dans un contexte impeccablement républicain. Citons les Detroit Lions, les Oakland Raiders, les Tampa Bay Buccaneers, ou les San Diego Chargers (les Destriers) pour constater une certaine cohérence des appellations où reviennent régulièrement les animaux sauvages, les hommes redoutables ${ }^{15}$ et les objets rapides, en référence à un passé plus ou moins mythique ${ }^{16}$. L'icône peut avoir plusieurs faces. Les New England Patriots ou les San Francisco Fortyniners rappellent l'héritage

14. « les bêtes les plus prédatrices et les oiseaux de proie » TCL, p.11.

15. L'équipe de la ville de Buffalo est un cas d'espèce avec un jeu de mot facile à la clé (Buffalo Bills)

16. Sur la totalité des équipes de la National Football League, les deux tiers des appellations entrent incontestablement dans cette catégorie (Broncos, Chargers, Chiefs, Raiders, 
historique ${ }^{17}$, les Pittsburgh Steelers la tradition industrielle. L'observation vaudrait aussi pour le football universitaire : les West Virginia Mountaineers se souviennent de la géographie de cet état «merveilleux et sauvage ${ }^{18} \gg$ à travers leur mascotte, un trappeur qui arbore un long et antique fusil de chasse. Dans ces exemples, l'affichage de la masculinité brutale et ancienne est quelque peu édulcoré, mais la tendance observée reste la même, à l'issue d'un décodage un peu plus attentif auquel nous invitons le lecteur.

La marque sportive la plus connue, Nike, s'est dotée d'un symbole dont l'histoire est bien connue. La société qui porte le nom assez peu martial de Blue Ribbon jusqu'en 1978 a un comportement assez classique quand elle baptise, par exemple, un de ses premiers modèles de chaussure la «Cortez». L'allusion directe au conquérant brûleur de vaisseaux va faire place à une stratégie de communication dans laquelle le thème de l'agression disparaît du niveau explicite. Un décodage devient nécessaire parce que le nom de la déesse de la victoire est en grec ancien, mais, plus encore, parce que le symbole adopté brouille les pistes. Le fameux swoosh, dessiné en 1971 par une étudiante en publicité de Portland, est depuis rentré dans la légende notamment parce qu'il n'aurait rapporté que 35 dollars à sa conceptrice, mais personne ne sait exactement ce qu'il représente. Que l'on y voie l'aile de la déesse ou un boomerang, l'imagination perçoit le concept plus que l'image de la vélocité, dont les aspérités agressives ont été prudemment gommées.

A sa création, le swoosh est une forme plaisante mais énigmatique qui prend du sens uniquement lorsqu'il est accolé au nom de la firme. De 1971 à 1985, le dessin sert à souligner le nom, à mettre en valeur les quatre lettres du mot Nike, avant de prendre son autonomie, au fil des ans et au gré des nouvelles maquettes, pour finalement se substituer au nom de la firme. Le swoosh a remplacé les quatre lettres ; il est devenu directement lisible sur toute la planète.

L'allusion au Panthéon de la Grèce antique était déjà extrêmement lointaine. L'éclipse progressive du nom de la déesse pour faire place au pur symbole souligne le triomphe ultime des efforts de marketing de la firme. Elle a réussi à imposer une reconnaissance visuelle instantanée, c'est-à-dire le logo à la place du logos. Mais cette permutation dégage un

Colts, Jaguar, Titans, Patriots, Rams, Seahawks, Bears, Lions, Vikings, Buccaneers, Falcons, Panthers, Cowboys, Eagles, Giants, Redskins) mais presque tous les autres possèdent par métonymie une dimension rapide ou redoutable (Packers, Jets, Dolphins, Ravens, Steelers, etc.) à l'exception peut-être des New Orleans Saints, qui relèvent de l'imagerie du Jazz.

17. Respectivement la Guerre d'Indépendance et la Ruée vers l'Or de 1849.

18. Wild Wonderful West Virginia est la devise de l'état de Virginie occidentale. 
espace pour un discours dédoublé. Elle permet aussi de saisir comment le discours explicite de la firme sur elle-même coexiste avec d'autres formes d'affirmation de son identité. C'est cette dernière dimension qui constitue l'implicite que nous cherchons à ramener au jour.

À ce stade, nous avons progressé dans la caractérisation des deux faces du sport, identifiant tour à tour une tendance explicitement démocratique et une implicite dont la théorie de Veblen permet de discerner les contours. Mais pour se persuader de l'unicité de notre objet d'étude, il est nécessaire de réintroduire dans le tableau la variable temps.

Le sport, la pratique comme le mot qui la désigne, a en effet une histoire spécifique, qui permet de distinguer un présent civilisé d'un passé barbare, relativement proche chronologiquement, et qui survit peut-être sur le versant implicite de cette réalité.

Il faut noter que la définition du mot sport varie assez fortement au $\mathrm{XIX}^{\mathrm{e}}$ siècle. Dans la plupart des langues parlées actuellement, le mot anglais a prévalu pour désigner le sport au sens moderne du terme. Le mot n'est pourtant pas originellement anglo-saxon puisqu'il vient de l'ancien français déport ${ }^{19}$ qui est reconnaissable dans le mot espagnol deporte et le verbe anglais to disport. Le français a dû réimporter le mot en 1828 , à un moment où sa signification a changé. Signalons, pour le mot anglais, trois faisceaux de sens qui sont également intéressants pour notre propos :

- sport au sens ancien de bloodsport, c'est-à-dire le passe-temps de la noblesse, qui se partage entre chasse et guerre, le premier servant à s'aguerrir pour la seconde;

- l'expression «to be a good sport» désigne le joueur loyal et bon perdant, pour qui l'essentiel est - selon la formule célèbre - de participer;

- le verbe «to sport» signifie arborer, par exemple, un vêtement neuf.

Le glissement de sens entre un passé relativement sanguinaire et une acception beaucoup plus en accord avec la sensibilité moderne se mesure entre la première et la seconde définition, respectivement point de départ et d'arrivée de cette évolution du mot. Le troisième sens nous renvoie à la partie ostentatoire dont nous cherchons à éclairer la portée par les emprunts à Veblen. Ainsi le sport est peut-être une activité physique relativement facile à décrire, mais il semble que la représentation du sport ne puisse échapper à la complexité. Dès l'origine, les peintures de Lascaux (1500 à 1300 avant J.-C.) décrivent les techniques de la chasse, mais dans le même temps, nous livrent une esthétique qui nous donne fugacement accès à la pensée magique.

19. Se déporter signifie alors s'amuser. 
De Lascaux à Coubertin, le sens du mot s'est infléchi dans de telles proportions qu'il est impossible de ne retenir que l'acception hygiéniste et bien-pensante qui prévaut désormais sans rechercher les échos d'un passé relativement proche. La grande période de basculement pour les Iles Britanniques se situe effectivement au XIX siècle, avec la création des premières sociétés protectrices des animaux qui défendent les droits des animaux d'élevage. La Société pour la prévention de la cruauté envers les animaux (Society for the Prevention of Cruelty to Animals) est fondée en 1822 et reçoit le soutien officiel de la couronne en $1840^{20}$. Mais il faudra attendre 1924 pour que la Ligue contre les sports cruels (League against Cruel Sports ${ }^{21}$ ) prenne spécifiquement la défense du gibier. La forme la plus emblématique, la chasse au renard avec mise à mort, ne sera interdite qu'en 2002 en Ecosse et en 2004 en Angleterre et au Pays de Galles. Des divertissements sanglants comme les diverses formes de baiting, c'est-à-dire les combat d'animaux entre eux, mais aussi quelquefois entre homme et animal, dont le plus célèbre est le bearbaiting (ours contre chiens), disparaissent à l'aube du $\mathrm{XX}^{\mathrm{e}}$ siècle. En revanche, la pratique du Teastas Mor, c'est-à-dire la certification des chiens terriers à l'issue d'un combat contre un blaireau, est pratiquée - avec des règles très strictes jusqu'en 1968 par le Irish Kennel Club. La sensibilité moderne condamne fermement, avec lourdes amendes à la clé, ce genre de pratique qualifiée «d'activité barbare du dix-septième siècle qui n'a pas sa place dans la Grande Bretagne du vingt-et-unième siècle ${ }^{22} \gg$. Mais les bloodsports ne sont pas uniquement une sorte de survivance barbare, dans la mesure où des pans entiers du secteur, tout à fait florissants, relèvent clairement de cette tradition.

\section{Permanence d'un implicite}

Les firmes qui fabriquent et commercialisent des armes à feu de loisir se prêtent de façon assez prévisible à la grille d'analyse de Veblen. Sans prétendre rendre compte de manière exhaustive des sites web des armuriers britanniques et américains, nous citerons quelques exemples dont la représentativité nous semble facilement vérifiable par le lecteur.

Au-delà des évidences, notons que Veblen permet d'établir une typologie relativement fine entre la présentation habituelle en Grande-

20. Elle devient RSPCA où le R correspond à royal. L'équivalent américain, American Society for the Prevention of Cruelty to Animals date de 1866.

21. Voir le site très complet de la League Against Cruel Sports pour les détails.

22.Déclaration d'un responsable de la $R S P C A$ en 2005 à l'issue d'un procès pour Badger Baiting près de Nottingham : voir le site de Sheffield Today. 
Bretagne et celle qui prévaut aux Etats-Unis. La firme britannique Holland \& Holland, fondée en 1835, par exemple, s'adresse à la classe de loisirs, c'est-à-dire aux héritiers assagis et néanmoins prestigieux d'une tradition violente. Le site décrit le style aristocratique du fondateur amateur de chasse à la grouse (tétras) dans le Yorkshire, et met en ligne la galerie de gravures animalières qui rappelle discrètement le cadre de vie de la gentry $^{23}$. L'animal transformé en trophée n'est plus le corps sanglant dans le carnier mais une œuvre d'art sous verre. L'entreprise diffuse ainsi une image des armes de chasse qui met à distance la mise à mort du gibier, et dépeint de façon un peu convenue la vie du manoir. Notons que, dans les années 1990, Holland \& Holland lance une ligne de vêtements au classicisme de bon aloi qui cadre parfaitement avec la tonalité d'ensemble.

Aux Etats-Unis, les armuriers semblent adopter une stratégie de présentation de leurs produits qui mette moins l'accent sur la distinction, ce qui est relativement prévisible dans une société qui se veut sans classes sociales. Lorsque la société Bill Hanus Birdguns de l'Oregon, par exemple, présente en ligne sa gamme de produits, il est clair que la toile de fond suggérée est un décor rustique qui n'a pas la sophistication des concurrents d'Outre-Atlantique. Le site montre le fondateur éponyme, souriant grand-père myope et moustachu, au visage rond, qui porte une chemise à carreaux. La distanciation vis-à-vis de la violence passe par la technicité, qui confère une sorte de magie irréelle au «monde merveilleux du calibre 16, 20 et $28 »$ («The Wonderful World of 16, 20 and 28 Gauge»). Mais cette technicité peut facilement quitter les terres rassurantes de la ruralité souriante pour conduire le client potentiel vers un imaginaire visiblement plus guerrier, c'est-à-dire, du point de vue véblénien, encore plus archaïque.

La société Wilson («Wilson Custom Firearms and accessories since $1977 \gg$ ) arbore non seulement un logo en forme d'aigle, mais la couverture de son catalogue $2006^{24}$ s'orne de trois armes qui évoquent clairement la guerre plus que la chasse : un fusil d'assaut, un pistolet automatique et un riot gun sur fond de paysage littoral. La visite du site de Wilson firearms nous livre bientôt la vision incongrue et légèrement inquiétante d'un homme que la moustache et l'âge désigneraient - une fois encore - comme un paisible grand-père. Mais le personnage porte un casque lourd, un harnachement et un treillis de combat, un gilet pareballes et pour faire bonne mesure brandit un fusil d'assaut. Le doute

23. Catégorie sans équivalent réel dans l'Ancien Régime en France, la gentry est constituée de roturiers enrichis à qui la monarchie donne accès à la petite noblesse afin d'en faire des soutiens du régime.

24. Voir la page d'accueil du site Wilson. 
n'est pas permis à l'examen d'un tel tableau : le loisir dont on nous vante ici les mérites ressemble plus à la guerre qu'à la chasse. Du point de vue de Veblen, d'ailleurs, la distinction entre les deux activités n'est pas pertinente, puisque «le guerrier et le chasseur récoltent ce qu'ils n'ont pas semé» (Veblen, TCL, p. 9). Si l'on cherche des causes objectives, à la différence de présentation, il faut bien sûr admettre que la législation sur les armes en vigueur aux Etats-Unis, nettement moins contraignante qu'en Grande-Bretagne, permet la commercialisation de tels produits malgré des restrictions introduites récemment. Mais ce qui est frappant, c'est qu'en dépit du caractère archaïque au sens de Veblen, nous sommes en présence d'un imaginaire tout à fait adapté à la modernité. Les clients potentiels qui visitent le site web de l'entreprise sont invités à «remplir leur caddie» exactement comme pour n'importe quel achat en ligne, en cliquant sur les articles désirés.

Les commentaires des clients cités dans la rubrique Customers' testimonials sont louangeurs, ce qui est attendu. Ce qui l'est moins, en revanche, c'est qu'ils traitent l'arme à feu comme un produit absolument banal. «You make the best product on the market without question» déclare ainsi un consommateur satisfait. Sans le contexte, il serait impossible de deviner la nature si particulière du produit et du marché. L'euphémisation passe par une référence au modèle économique, ce que confirme un autre commentaire qui vante la grande qualité artisanale (quality of workmanship) et le service au consommateur (outstanding customer service). Le terme workmanship est frappant ici parce que dans le système de Veblen, l'instinct artisanal (instinct of workmanship), s'oppose très exactement à l'instinct de prédation. Pour le Veblen théoricien de la firme, le premier caractérise la classe des ingénieurs et des techniciens ${ }^{25}$ tandis que la prédation se retrouve dans la conduite des «capitaines d'industrie» qui génèrent de la plus-value financière en instrumentalisant les crises cycliques qui perturbent la production. Autrement dit, la lecture selon la grille véblénienne met ici en relief une opposition de type historique entre un explicite qui emprunte ses thèmes et son lexique au monde de l'entreprise et au modèle du marché, et un implicite à la fois pré-capitaliste et caractéristique de la forme la plus récente, celle du capitalisme financier que Veblen est l'un des premiers à décrire clairement.

L'évolution à long terme dira si l'implicite sulfureux qui transparaît est voué à disparaître, selon un scénario que décrit, en le déplorant, Nietzsche pour qui «le sens de toute culture [est] de domestiquer le fauve 'humain' pour en faire l'élevage, un animal apprivoisé et civilisé

25. Une caractéristique à rapprocher de l'éthique protestante du travail. 
[...] (Nietzsche, p. 53). Sauf retour improbable des «monstres triomphants» (Nietzsche, p. 51), le citoyen civilisé se contentera donc vraisemblablement d'acheter les produits des équipementiers, et à consommer des spectacles sportifs, presque par substitution, parce que ces loisirs constituent le seul lien avec l'univers révolu de la prédation, dont on ne peut ouvertement se réclamer.

Les rapports du sport avec la violence en général sont extrêmement bien documentés. En tracer un tableau, depuis les émeutes sanglantes pendant les courses de char de Constantinople en 532 jusqu'à la tragédie du Heysel en $1985^{26}$ serait fort long mais permettrait de mettre à jour une certaine continuité historique, et une permanence actuelle. La figure de l'athlète entretient toujours des rapports complexes avec celle du héros mais aussi du criminel. Les noms de la patineuse Tonya Harding, du boxeur Mike Tyson, de Gustavo Kuerten ou d'Eric Cantona viennent spontanément à l'esprit à cause de la couverture médiatique dont leurs incartades plus ou moins graves ont bénéficié. La palme de ce triste palmarès revient sans doute à O.J. Simpson que la justice californienne finit par proclamer responsable mais non coupable d'une double meurtre à l'issue de deux procès interminables.

Les exemples rapidement évoqués précédemment n'entendent pas démontrer que l'athlète violent est au-dessus des lois, mais si l'on dépouille la masse des articles évoquant l'inconduite des athlètes américains sur les terrains et sur les campus, force est de constater «qu'il y a une norme dans les universités pour l'équipe de football [américain] et une autre pour les garçons catalogués comme "ordinaires" (unpopular) ${ }^{27}$ ». Si l'on applique le schéma de Veblen sur le caractère archaïque des normes qui régissent notre réaction à de tels cas, une certaine logique se dessine. Elle permet de saisir pourquoi un athlète violent de l'équipe des Badgers de l'université du Wisconsin est explicitement condamnable ceci ne souffre évidemment aucune discussion ${ }^{28}$ - mais son acte semble se situer sur un point aveugle de la perception morale. La coïncidence avec l'imaginaire totémique et initiatique décrit, entre autres par Eliade ${ }^{29}$, éveille, dans ce genre de cas, un écho troublant.

On nous objectera fort justement que toute violence ne relève pas de la prédation. Mais l'attitude ambiguë du corps social vis-à-vis du prédateur lorsqu'il est athlète de haut niveau nous révèle que nous approchons

26. Des supporters de Liverpool FC envahirent les tribunes de la Juventus, causant un bousculade où 39 personnes perdirent la vie.

27. Glenda Katz-Felder : voir article en ligne.

28. Détails dans l'article de Joanna Salmen sur le site du Badger Herald.

29. Nous renvoyons au chapitre V (p. 181-223) de Mircea Eliade. 
d'un point d'inflexion du jugement moral. Dans ce cas, la prédation constitue une transgression éminemment condamnable, mais presque nécessaire dans la mesure où elle enclenche le mécanisme d'expulsion qui va refonder la cohérence du corps social. La vénération s'inverse en réprobation; le héros devient alors victime expiatoire. Cette disgrâce de l'athlète, exclu après avoir été pratiquement divinisé nous renvoie à la théorie développée par René Girard à partir des années soixante-dix ${ }^{30}$. L'articulation entre la grille de Veblen et celle de Girard pourrait se schématiser de la façon suivante :

\begin{tabular}{llcccc}
\hline $\begin{array}{l}\text { Corps social } \rightarrow \text { Athlète } \\
\text { (simples mortels) }\end{array}$ & $\rightarrow$ Prouesse & $\rightarrow \begin{array}{l}\text { Statut de héros } \\
\text { (semi-divin) }\end{array}$ & $\rightarrow \begin{array}{l}\text { Transgression } \\
\text { Prédatrice } \\
\text { (répulsion/prestige) }\end{array}$ & $\begin{array}{l}\text { Victime } \\
\text { Expiatoire }\end{array}$ \\
$\begin{array}{l}\text { Retour à la } \\
\text { cohésion }\end{array}$ & $\leftarrow$ & $\leftarrow$ & $\leftarrow$ & $\leftarrow$ \\
& Zone de pertinence de Veblen
\end{tabular}

Schéma 1- Articulation Veblen / Girard

Un meurtre aussi choquant que celui d'Andrés Escobar ${ }^{31}$ s'inscrit $^{\prime}$ dans un tel schéma, qui explique aussi la fascination «cathartique» (emotional cleansing ${ }^{32}$ ) suscités par l'affaire Simpson.

\section{Les règles de la création de valeur immatérielle}

Le discours explicite des grands équipementiers sportifs s'avère en définitive relativement complexe. Sur un fond consensuel, égalitariste et bien-pensant dans une société du politiquement correct, finissent par se détacher les contours fuyants d'un implicite archaïque, violent, aristocratique et provoquant, voire transgressif. Le mérite d'un détour par la sémiotique de Veblen est de permettre de nommer de façon synthétique l'implicite qui se situe aux limites de notre observation. L'exercice de communication, dès lors, ne peut que chercher à concilier deux messages incompatibles, voire diamétralement opposés. Le groupe Nike résume le grand écart que sa stratégie lui impose. Le groupe doit s'attacher la caution d'un athlète en vue - tel que Michael Jordan - par des contrats très généreux. La technologie des semelles à coussin d'air, qui avait donné le

30. Le texte majeur de Girard sur cette question est La Violence et le sacré.

31. Ce malheureux footballeur colombien marque contre son camp et assure la victoire de l'adversaire. Peu après, un supporter le tue de six coups de feu en ponctuant chaque détonation du cri « but ! ».

32. Expression du Dr Richard Lustberg : voir le site de Psychology of Sports. 
modèle Air Force One $e^{33}$ prendra le nom du célèbre basketteur et connaîtra un succès remarquable, précisément parce que les simples mortels seront très nombreux à l'acheter. C'est là que réside la tension au cœur du message du groupe Nike. L'un de ses fondateurs, Bill Bowerman, s'était rendu célèbre lorsqu'il n'était encore qu'un entraineur à l'université d'Oregon par la formule : «If you have a body, you are an athlete ${ }^{34}$ ». Le lecteur en déduira avec raison que seuls les purs esprits ne sont pas pour Nike des clients potentiels. On ne sait s'il faut saluer l'ardeur démocratique ou savourer l'humour involontaire d'un tel programme qui met l'exceptionnel héroïque à la portée de tous.

Toutefois, cette formulation est moins paradoxale qu'il n'y paraît car le business model des équipementiers sportifs diffère fondamentalement de celui des produits de luxe dont la diffusion est nécessairement limitée par une politique de prix dissuasive et sélective. Pour les groupes de sport, cette stratégie consiste à augmenter la rentabilité visant un marché de masse sans jouer la baisse des prix de détail. La gageure consiste à faire payer une sorte de prime à l'imaginaire pour des achats qui offrent implicitement aux masses l'accès à la classe de loisirs, ou plutôt à ses représentations. Le discours implicite, bien-pensant, est présent pour rendre l'acte d'achat compatible avec les censures sociales, car il est bien évident qu'un marketing explicite de l'agression et de la domination serait contre-productif.

La connivence entre le marchand et l'acheteur dépasse ici le cadre de la transaction courante sur le marché des biens. C'est pourquoi nous ne souscrirons pas aveuglément aux thèses conjointes de l'hypocrisie manipulatrice des firmes et de la naïveté du consommateur. Réconcilier l'inconciliable - l'élitisme et la consommation de masse - permet de convaincre le consommateur de surpayer un produit dont il a objectivement assez peu l'usage. Pour vendre un produit dix ou vingt fois plus cher que sa valeur-travail, Veblen dirait qu'il faut lui incorporer l'imaginaire sulfureux de la classe de loisirs, en l'intégrant à la stratégie de communication, nécessairement sur le mode subliminal, et donc implicite.

Notre étude ne cherche pas à infirmer le postulat de rationalité du sujet économique, pilier de la doctrine libérale. Mais le détour par la théorie institutionnaliste, et tout particulièrement celle de Veblen, nous semble de nature à rendre compte de façon convaincante des deux niveaux, explicite et implicite, du message émis, et de sa réception par un consommateur doté de mémoire et de profondeur psychologique.

33. C'est-à-dire l'avion présidentiel aux Etats-Unis. L'appellation est de 1982.

34. La citation et l'analyse sont sur le site de la Western Michigan University. 


\section{Références bibliographiques}

Bruckner Pascal, La Mélancolie démocratique : Comment vivre sans ennemis? Seuil, 1990.

Ehrenberg Alain, «Des stades sans dieux» in Bruckner, p. 86 [Le Débat, n40].

Eliade Mircea, Initiations, rites, sociétés secrètes, Gallimard, 1959.

Girard René, La Violence et le Sacré, Grasset, 1972.

Nietzsche Frédéric, La Généalogie de la morale, Mercure de France, 1964.

Katz-Felder Glenda, «Teenage Player is from Sayville» sur site: $<$ www.geocities.com/sayswamp/football.htm>

Veblen Thornstein, The Theory of the Leisure Class, Mineola, NY : Dover, 1994 [New York : Macmillan, 1899].

—, The Theory of Business Enterprise, 1904. Texte sur site McMaster : <http://socserv2.socsci.mcmaster.ca/ econ/ugcm/3113/veblen/busent>

\section{Sites Web cités (consultables au 30 mai 2007)}

Adidas: <http://www.adidas-group.com/en/sustainability/welcome.asp>.

Badger Herald : <http://badgerherald.com/news/2004/a/30/uw_football_player_a.php>

Bill Hanus Birdguns: $<$ http://www.billhanusbirdguns.com>

Billings Gazette :

<http://www/billingsgazette.com/newdex.php? display=rednews/2005/04/03/build/business/65-mother-boyle.inc>.

League Against Cruel Sports $<$ http://www.league.uk.com>

Nike: <http://www.nike.com/nikebiz/nikefoundation/who/jhtml>.

Psychology of Sports: «An analysis of current topics and issues in Sport», sept. 1995 sur le site de la revue: <http://www.psychologyof sports.com/couch/couch09_1995.htm>.

Reebock Human Rights Foundation: <http://reebok.com/static/global/initiatives/rights/pdf/RHRF_Annual_Report_2003.pdf>

Sheffield Today : «Badger baiter banned after terriers hurt» Sheffield Today, 8 août 2005 :

< http://sheffieldtoday.net> article $\mathrm{n}^{\circ} 1108258$.

Western Michigan University : $<$ http://wmich.edu/j1evansk/bus270.htm>

Wilson : $<$ http://www.wilson.com>.

Yogi Berra Museum : $<$ http://www.yogiberramuseum.org> 